\title{
ARTICLE The susceptibility to chronic social defeat stress is related to low hippocampal extrasynaptic NMDA receptor function
}

\author{
Yiu Chung Tse ${ }^{1}$, Joëlle Lopez ${ }^{1}$, Alexandre Moquin ${ }^{2}$, Shui-Ming Alice Wong ${ }^{1}$, Dusica Maysinger ${ }^{2}$ and Tak Pan Wong ${ }^{1,3}$
}

\begin{abstract}
$\mathrm{N}$-methyl-D-aspartate receptors (NMDARs) have been highly implicated in the pathogenesis and treatment of depression. While NMDARs can be found inside and outside glutamate synapses, it remains unclear if NMDARs at synaptic (sNMDAR) and extrasynaptic locations (exNMDAR) play different roles in the formation of depression-related behaviors. Using chronic social defeat stress (CSDS), an animal model for anxiety- and depression-related behaviors, we found that mice susceptible to CSDS exhibited low hippocampal exNMDAR function. Raising exNMDAR function by enhancing the release of glutamate from astrocytic cystineglutamate antiporters or targeting extrasynaptic receptors with agonist-coated gold nanoparticles that cannot enter the synaptic cleft prevented social avoidance behavior in stressed mice. Interestingly, ketamine, which is a fast-acting antidepressant, exhibited stronger blockade to sNMDARs than to exNMDARs. These findings suggest that the susceptibility and resilience of mice toward CSDS is related to low and high exNMDAR function in the hippocampus, respectively. Enhancing exNMDAR function could be a novel treatment approach for mood and anxiety disorders.
\end{abstract}

Neuropsychopharmacology (2019) 44:1310-1318; https://doi.org/10.1038/s41386-019-0325-8

\section{INTRODUCTION}

Aside from its well-known roles in neuronal fate and synaptic plasticity, $\mathrm{N}$-methyl-D-aspartate receptors (NMDARs) have been implicated in the pathogenesis and treatment of depression. Not only do binding studies reveal changes in NMDAR expression in brain tissue from depressed patients [1], but several NMDAR antagonists, in particular ketamine, also exhibit antidepressant-like properties [2]. NMDARs can be found within (synaptic, sNMDAR) and outside synapses (extrasynaptic, exNMDAR). NMDARs in synaptic and extrasynaptic locations exert different effects on neuronal fate and synaptic plasticity via distinct signaling pathways $[3,4]$. These findings beg the question of whether sNMDARs and exNMDARs play different roles in depression.

A concurrent view in the field suggests that exNMDAR activation underlies some depression symptoms [5]. exNMDAR activation could be facilitated by impaired glutamate uptake after the loss of astrocytes [6]. Moreover, exNMDARs have been associated with synaptic pruning [4], which could be responsible for spine loss in depression [7]. Finally, antidepressant effects can be produced by some specific antagonists for GluN2B-containing NMDARs [8], which are likely enriched in extrasynaptic locations. Nonetheless, direct evidence to support a contribution of exNMDARs to depression symptoms in humans or to depression-related behaviors in animal models is lacking.

Chronic social defeat stress (CSDS) has been used to examine mechanisms underlying the pathogenesis of anxiety- and depression-related behaviors [9]. In this model, mice are stressed by multiple episodes of social defeat and screened for their performance in a social interaction test ( $\mathrm{SI}$ test). Mice that are susceptible to this social stressor show avoidance behavior in the
SI test and other depression-related behaviors such as anhedonia, behavioral despair, and sleep disturbance [9-11]. Some of these depression-related behaviors are sensitive to classical (e.g. fluoxetine [12]) and fast-acting (e.g. ketamine [13]) antidepressants. However, resilient mice do not show these behaviors after stress. Mice with different susceptibilities to CSDS have been used to reveal biological mechanisms of stress susceptibility and resilience, which could have important implications for the vulnerability to depression and anxiety in humans. Our objective is to differentiate the contribution of SNMDAR vs. exNMDAR function to the susceptibility to CSDS. We focused on the dorsal hippocampus, which has not only been highly sensitive to chronic stressors, but also shown to be related to stress susceptibility [14-16]. We showed that not only low hippocampal exNMDAR function is associated with stress susceptibility, enhancing exNMDAR function prevented social avoidance behavior after CSDS. To our knowledge, this is the first study to reveal a regulatory role of exNMDARs in stress susceptibility.

\section{MATERIALS AND METHODS}

Animals

All care and use of animals were in accordance with the Canadian Council on Animal Care guidelines (protocol number: 2010-5935). Adult male $\mathrm{C} 57 \mathrm{BL} / 6 \mathrm{NCrl}$ (C57) mice (8-12-week-old) and male CD1 retired breeders (6-8-month-old) were obtained from Charles River Laboratories. They were housed individually in the Douglas Institute animal facility and maintained on a 12/12 light cycle (light on: 8AM). Food and water were available ad libitum.

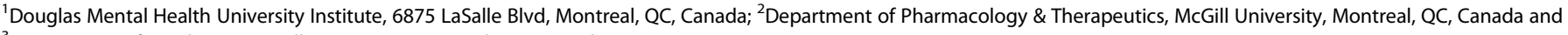
${ }^{3}$ Department of Psychiatry, McGill University, Montreal, QC, Canada

Correspondence: Tak Pan. Wong (takpan.wong@mcgill.ca)

These authors contributed equally: Yiu Chung Tse, Joëlle Lopez
}

Received: 16 July 2018 Revised: 16 January 2019 Accepted: 19 January 2019

Published online: 25 January 2019 


\section{Drugs}

Unless indicated otherwise, all reagents were obtained from Sigma-Aldrich. Ketamine was obtained from Toronto Research Chemicals (Health Canada Authorization 39827.04.16). dl-Threo- $\beta$ benzyloxyaspartic acid (TBOA) and RS-tetrazol-5-yl glycine (TZG) were obtained from Tocris. Gold chloride trihydrate was obtained from Alfa Aesar. a-Methoxy- $\omega$-mercapto poly(ethylene glycol) $\left(\mathrm{H}_{3} \mathrm{C}-\mathrm{O}-\mathrm{PEG}-\mathrm{SH}, 2000 \mathrm{~g} / \mathrm{mol}\right)$ and carboxy- $\omega$-mercapto poly(ethylene glycol) (HOOC-PEG-SH, $3400 \mathrm{~g} / \mathrm{mol}$ ) were obtained from Creative PEGWorks.

Drug-coated gold nanoparticles (GNP)

GNP synthesis. CTAB (hexadecyltrimethylammonium bromide) covered spherical gold nanoparticle (GNPs) $(d=14 \mathrm{~nm})$ were prepared in water by adding an ice-cold solution of sodium borohydride $(0.500 \mathrm{ml}, 0.01 \mathrm{M})$ to $\mathrm{HAuCl}_{4} \cdot 3 \mathrm{H}_{2} \mathrm{O}(0.125 \mathrm{ml}, 0.01 \mathrm{M})$ and CTAB $(4.375 \mathrm{ml}, 0.075 \mathrm{M})$ [17] for $2 \mathrm{~h}$. The seed solution $(0.1$ $\mathrm{ml}$ ) was added to a mixture of CTAB $(1.6 \mathrm{ml}, 0.10 \mathrm{M}), \mathrm{HAuCl}_{4} \cdot 3 \mathrm{H}_{2} \mathrm{O}$ $(2 \mathrm{ml}, 0.01 \mathrm{M})$, and ascorbic acid solution $(0.6 \mathrm{ml}, 0.10 \mathrm{M})$ in $90 \mathrm{ml}$ water. Immediately upon addition of the seed solution, the mixture was mixed gently for $10 \mathrm{~s}$ and left undisturbed for $>3 \mathrm{~h}$. GNPs were pelleted by centrifugation $(3000 \mathrm{~g}, 1 \mathrm{~h})$ and resuspended in water $(5 \mathrm{ml})$. The solutions were kept at $37^{\circ} \mathrm{C}$ to prevent $C T A B$ crystallization.

GNP functionalization. GNPs were functionalized with memantine and TZG according to Savchenko et al. [18] with slight modifications. Briefly, the CTAB shell was displaced by a mixture of thiol-modified PEGs terminated with methoxy or carboxyl, at a nominal molar ratio of 1:1 methoxy:carboxyl. GNP concentrations were determined by absorbance, using the plasmon peak at 521 $\mathrm{nm}$ and an extinction coefficient of $6.313 \times 10^{8} \mathrm{M}^{-1} \mathrm{~cm}^{-1}$. To coat the particles, solutions of $\mathrm{H}_{3} \mathrm{C}-\mathrm{O}-\mathrm{PEG}-\mathrm{SH}(400 \mu \mathrm{l}, 24 \mathrm{mg} / \mathrm{ml}$ in ethanol) and HOOC-PEG-SH $(400 \mu \mathrm{l}, 40.8 \mathrm{mg} / \mathrm{ml}$ in ethanol) were premixed and combined with $100 \mathrm{ml}$ of GNP-CTAB. The following day, the GNP were pelleted three times $(18,000 \mathrm{~g}, 30 \mathrm{~min})$ and resuspended in water to remove free ligands. Coupling of drug molecules was performed in 2-( $\mathrm{N}$-morpholino)ethanesulfonic acid buffer (MES, $0.1 \mathrm{M}$, pH 4.3). First, $N$-(3-dimethylaminopropyl)- $N$ '-ethylcarbodiimide hydrochloride (EDC, $100 \mu \mathrm{l}$ in $0.16 \mathrm{M}$ in MES) was added to $300 \mu \mathrm{GNP}$ and incubated for $2 \mathrm{~min}$. Then $\mathrm{N}$ hydroxysuccinimide (NHS, $100 \mu \mathrm{l}$ in $0.26 \mathrm{M}$ MES) was added, incubated for $5 \mathrm{~min}$, followed by the amine-containing drug (100 $\mu l, 0.07 \mathrm{M}$ in water). After a $1 \mathrm{~h}$ coupling reaction, carbonate buffer ( $25 \mu \mathrm{l}, 7.5 \%$ solution, $\mathrm{pH}$ 9.3) was added to raise the $\mathrm{pH}$ level. After $1 \mathrm{~h}, 50 \mu \mathrm{l}$ more of each of EDC and NHS solutions were added and reaction continued overnight. The GNP conjugates were twice pelleted $(18,000 \mathrm{~g}, 30 \mathrm{~min})$ and resuspended in $1 \times$ PBS-T $(0.005 \%$ Tween $20 \mathrm{w} / \mathrm{w}$ ), and stored at $4{ }^{\circ} \mathrm{C}$ overnight followed by dialysis in $1 \times$ PBS using a $20 \mathrm{kDa}$ Slide-a-Lyzer (Pierce), with three buffer changes over $48 \mathrm{~h}$. The memantine- and TZG-coated nanoparticle products were diluted in PBS and filtered $(0.45 \mu \mathrm{m})$. The Z-average hydrodynamic diameter of drug-coated GNPs is $31 \mathrm{~nm}$, a targeted size that is bigger than the estimated width of synaptic cleft $(\sim 15$ $\mathrm{nm}$ [19]).

Intrahippocampal drug infusions

Surgery. One week before the start of CSDS, C57 mice were anesthetized with isoflurane and placed on a stereotaxic apparatus. Stainless steel 26-gauge cannulae were bilaterally implanted in the dorsal hippocampus (AP: $-1.85, \mathrm{ML}: \pm 1.25$, DV: -1.5 ). The cannulae were maintained in place by dental cement which was fixed to the skull with two screws and protected by obturators and dust caps to prevent blockage. Drug infusions were performed one week after surgery.

Infusion procedures. Mice were handled daily and habituated to dust cap removal and injector insertion before drug infusion.
Drugs or their vehicles were infused $30 \mathrm{~min}$ before the SI test (see below). A total volume of $0.5 \mu \mathrm{l} / \mathrm{side}(0.1 \mu \mathrm{l} / \mathrm{min})$ was infused bilaterally into the dorsal hippocampus using a microinfusionpump. The injectors were left in place for an additional $10 \mathrm{~min}$ to ensure proper drug diffusion.

Behavioral testing

Social defeat. All animals were stressed in the light cycle of the day between 8:00 and 12:00 [20]. During each CSDS session, a resident CD1 aggressor mouse was allowed to attack an intruder C57 mouse for up to 10 times within $5 \mathrm{~min}$. The defeated C57 mouse, which displayed subordinate posture and avoidance of the aggressor during defeat, was housed in the same cage as the aggressor for $24 \mathrm{~h}$ with a transparent perforated partition to prevent physical contact between them. During each of the 10 daily CSDS sessions, each C57 mouse was defeated by and housed with a new resident CD1 aggressor. Control C57 mice were only handled daily and housed in pairs in a cage with a perforated partition.

SI test. An SI test was performed $24 \mathrm{~h}$ after the last CSDS session in an open field $(45 \mathrm{~cm} \times 45 \mathrm{~cm})$, which contains a virtual interaction zone near the perforated enclosure (for the CD1 mouse) in the middle of the north side of the open field (see Fig. 2a). Although mice between the perforated enclosure have limited physical contact, they could interact with each other through auditory, olfactory, and visual stimuli (e.g. see [21]). Putting the aggressor into an enclosure also prevents aggressive interaction between mice and ensures that SI was initiated by the defeated mouse outside the enclosure. Each C57 mouse was placed into the open field for two consecutive trials of $2.5 \mathrm{~min}$ : the first trial with an empty enclosure and the second trial with a CD1 mouse in the enclosure. During the second trial, each C57 mouse was able to interact with the CD1 mouse through the perforated partition of the enclosure. To avoid the effect of previous exposure to a CD1 mouse on the mouse performance in the SI test, we paired a CD1 mouse from another defeat experiment with the tested mouse during the SI test to ensure these two mice have not previously encountered each other. An SI ratio was calculated by dividing the time mice spent in the interaction zone during the second trial by that during the first trial. Defeated mice with an $\mathrm{SI}$ ratio $>1$ and a second trial interaction time $>50 \mathrm{~s}$ were considered resilient, while all susceptible mice displayed an SI ratio of $<1$.

\section{Electrophysiology recordings}

Slice preparation, patch clamp recording and data analysis were performed as described [22]. All animals were sacrificed to make slices during the light cycle of the day between 8:00 and 12:00. Synaptic responses were evoked by stimulating the Schaffer collateral-commissural pathway and recorded from the stratum radiatum in the dorsal hippocampal CA1 region. Both $\mathrm{GABA}_{\mathrm{A}}$ and AMPA receptors were blocked to isolate the NMDAR-mediated component in evoked responses. Evoked NMDAR-mediated field excitatory postsynaptic potentials (fEPSPs) were recorded with a low $\mathrm{Mg}^{2+}(0.05 \mathrm{mM})$ perfusing solution. All recordings were performed at room temperature.

Statistical analysis

Data are presented as mean \pm SEM. Normality of data was examined using the Shapiro-Wilk test. The $n$ in behavioral and electrophysiology experiments refers to the number of animals and the number of recordings, respectively. For electrophysiology data, the number of animals used in each experiment was also listed. Student's $t$-tests were used for comparisons between two groups. Multiple-group comparisons were analyzed using ANOVAs followed by post hoc Tukey's tests (parametric), or using Kruskal-Wallis test followed by post hoc Wilcoxon tests (nonparametric). 


\section{RESULTS}

We isolated evoked exNMDAR currents from dorsal hippocampal CA1 neurons of adult C57 mice by first blocking evoked sNMDAR currents with MK801 (40 $\mu \mathrm{M}$, Fig. 1a). MK801-induced blockade of sNMDARs was long lasting (see inset in Fig. 1a). The lasting MK801 blockade allowed us to use TBOA, a glutamate transporter blocker, to trigger glutamate spillover for activating exNMDARs (see also [23]). TBOA was used at $20 \mu \mathrm{M}$, since we found no further increase in exNMDAR currents at higher concentrations (Figure S1). TBOArelated currents can be completely blocked by an NMDAR antagonist APV (Fig. 1a). Comparing the kinetics of evoked sNMDAR and exNMDAR currents revealed the expected slower rise time of exNMDAR currents (Fig. 1b). However, sNMDAR currents also exhibited a slower decay property when compared to exNMDAR currents. We next investigated the contribution of GluN2B subunit-containing NMDARs to SNMDAR and exNMDAR currents, which could be related to their decay properties. While GluN2B-containing NMDARs are believed to be predominantly present outside hippocampal synapses after development ([24], but also see [25]), we did not observe differences in the percent blockade of both the amplitude and the charge of sNMDAR and exNMDAR currents caused by a GluN2B antagonist Ro25-6981 (Fig. 1c). Our findings suggest that GluN2B-containing NMDARs contribute similarly to sNMDAR and exNMDAR currents in the hippocampal CA1 region of adult C57 mice.

We then examined changes in SNMDAR and exNMDAR currents in the CA1 region of the dorsal hippocampus in mice after CSDS. Using the SI test, defeated mice can be separated into susceptible or resilient mice (Fig. 2a and S3). Resilient mice also exhibited an SI ratio that was similar to non-stressed control mice. Next, we examined sNMDAR function by recording evoked NMDARmediated fEPSPs. Comparing the relationship between fiber volley amplitude and the slope of NMDAR-mediated fEPSP revealed similar input/output relationships in these three mouse groups (Fig. 2b), suggesting no change in sNMDAR function. To compare exNMDAR function between these three mouse groups (Fig. 2c), the extrasynaptic current from each neuron was normalized by its sNMDAR current recorded before MK801 blockade. We found that both the amplitude (Fig. 2d) and the charge (Fig. 2e) of TBOAinduced exNMDAR currents in susceptible mice were significantly smaller than those of resilient and control mice. We also compared the kinetics of sNMDAR and exNMDAR currents from these mouse groups. While we found that the rise time and decay time constant of sNMDAR currents were shorter and larger than those of exNMDAR currents, respectively, we observed no differences in these kinetic properties between mouse groups (Figure S2).

exNMDAR currents of mice with different stress susceptibilities were further examined. Glutamate spillover for exNMDAR activation can be induced by tetanus stimulation (Figure S4A). In parallel to our TBOA findings, the amplitude of tetanus-induced exNMDAR currents (normalized by SNMDAR currents that were recorded from the same neurons) from susceptible mice was smaller than that from other mouse groups (Figure S4B). Furthermore, we found that the normalized charge of tetanus-induced exNMDAR currents from susceptible mice was smaller than that from resilient mice (Figure S4C). Alternatively, ambient levels of glutamate have been shown to selectively activate exNMDARs [26], which can be revealed by changes in whole-cell currents after APV blockade. We found that APV-induced blockade of whole-cell currents in resilient mice was significantly larger than that in other mouse groups (Figure S5). Taken together, these findings suggest that susceptible and resilient mice display low and high hippocampal exNMDAR functions, respectively.

If low exNMDAR function is related to the social avoidance of susceptible mice, enhancing exNMDAR function could ameliorate this depression-related behavior. exNMDAR activation could be facilitated by $\mathrm{N}$-acetylcysteine (NAC), which induces glutamate release from the cystine-glutamate antiporter on astrocytes [27].
A

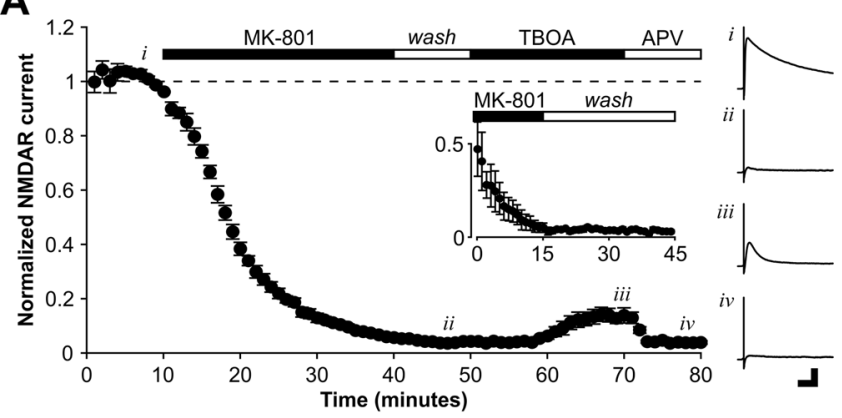

B
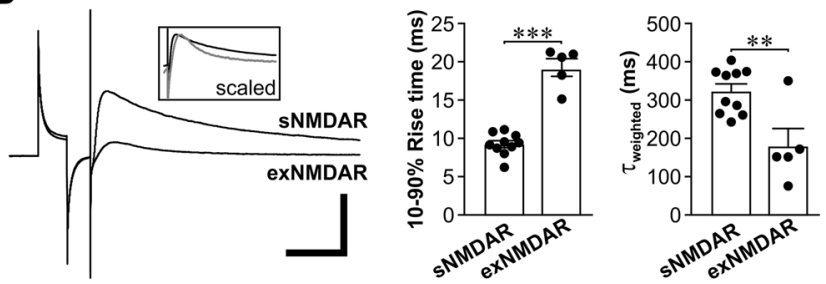

C
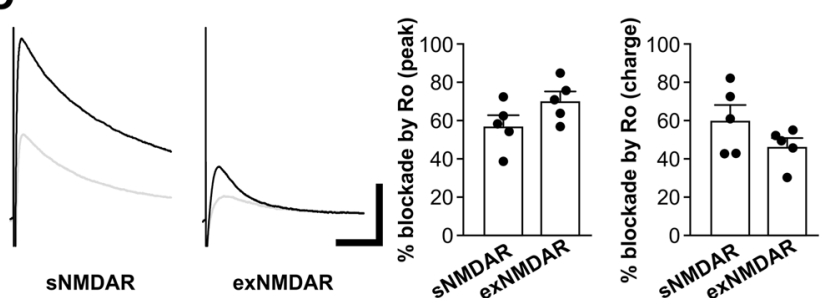

Fig. 1 Isolation of hippocampal extrasynaptic NMDAR currents. a Scatter plots ( $n=4$ neurons/4 mice) illustrate the time course for isolating extrasynaptic NMDAR (exNMDAR) currents. Recorded currents were normalized by synaptic NMDAR (sNMDAR) currents, (i). sNMDAR currents were blocked by MK801 $(40 \mu \mathrm{M}$, ii). exNMDAR currents were induced by TBOA $(20 \mu \mathrm{M}$, iii), which could be blocked by APV $(50 \mu \mathrm{M}$, iv). Traces on the right represent evoked NMDAR currents before (i) and after MK801 (ii), after TBOA (iii) and after APV treatments (iv). Inset: Scatter plot ( $n=4$ neurons/4 mice) showing the lasting MK801 blockade of NMDAR currents, which revealed no recovery during the $30 \mathrm{~min}$ long wash $(x$ and $y$ axis of this plot correspond to time (minutes) and normalized NMDAR current, respectively). Mean \pm SEM. Scale bar $=50 \mathrm{pA}, 100 \mathrm{~ms}$. b Left: Representative traces of SNMDAR (before MK801 blockade) and exNMDAR currents (in the presence of TBOA) that were recorded from a CA1 neuron. The part of the traces before exNMDAR currents are test pulses to show seal stability and stimulating artifacts. Inset shows the overlapping of SNMDAR (black) and peak-scaled exNMDAR traces (gray). Scale bar $=100 \mathrm{pA}, 100 \mathrm{~ms}$. Right: Histograms show the $10-90 \%$ rise time and decay time constant ( $\tau_{\text {weighted: }}$ weighted tau after double exponential fit). Rise time of sNMDAR: $9.24 \pm 0.46 \mathrm{~ms}$ ( $n=10$ neurons/10 mice) vs. rise time of exNMDAR: $19.2 \pm 1.16 \mathrm{~ms}$ ( $n=5$ neurons $/ 5$ mice); $t_{13}=9.76, p=2.37 \mathrm{E}-07$. Decay time constant of sNMDAR: $323.6 \pm 18.7 \mathrm{~ms}(n=10$ neurons $/ 10$ mice) vs. decay time constant of exNMDAR: $180.2 \pm 45.5 \mathrm{~ms} \quad(n=5$ neurons $/ 5$ mice); $t_{13}=3.50, p=0.004$. Mean \pm SEM, $* * p<0.01$, ${ }^{* * *} p<0.001$. c Representative traces of sNMDAR and exNMDAR currents before (black) and after (gray) the blockade produced by a specific GluN2B antagonist Ro25-6981 (Ro, $2 \mu \mathrm{M}$ ). Scale bar $=50 \mathrm{pA}$, $100 \mathrm{~ms}$. Histograms on the right show the percent Ro-induced blockade of the amplitude and the charge of sNMDAR and exNMDAR currents. Percent blockade of sNMDAR amplitude: $57.2 \pm 5.5 \% \quad(n=5$ neurons $/ 5$ mice $)$ vs. percent blockade of exNMDAR amplitude: $70.4 \pm 4.8 \%(n=5$ neurons $/ 5$ mice $) ; t_{8}=1.80$, $p=0.110$. Percent blockade of sNMDAR charge: $60.2 \pm 7.9 \%(n=5$ neurons/5 mice) vs. percent blockade of exNMDAR charge: $46.6 \pm$ $4.3 \%$ ( $n=5$ neurons $/ 5$ mice); $t_{8}=1.52, p=0.167$. Mean \pm SEM 
A
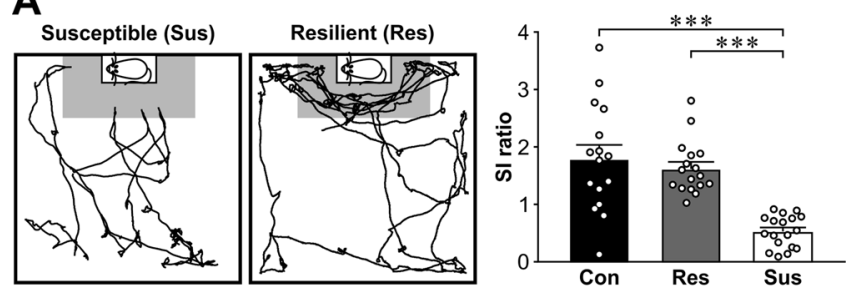

B
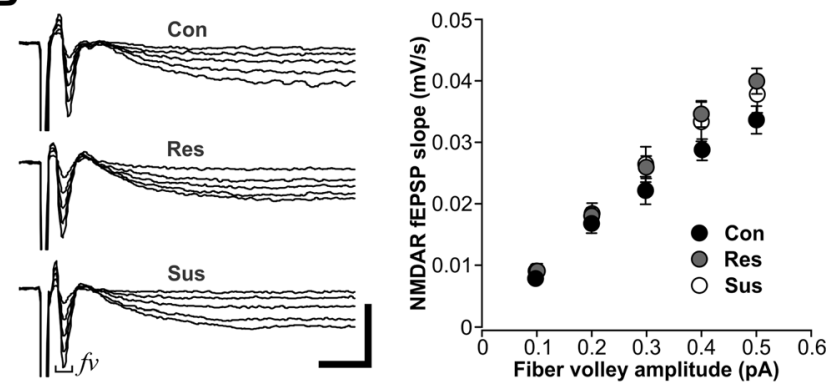

C

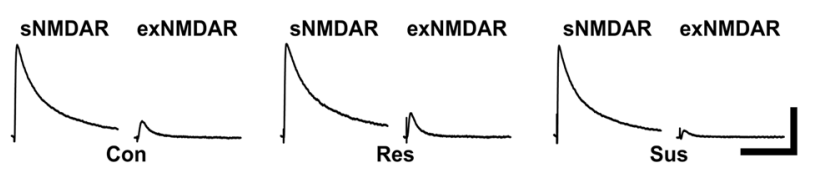

D

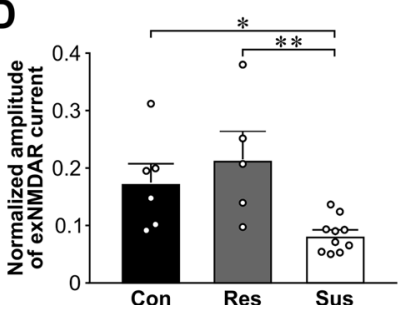

E

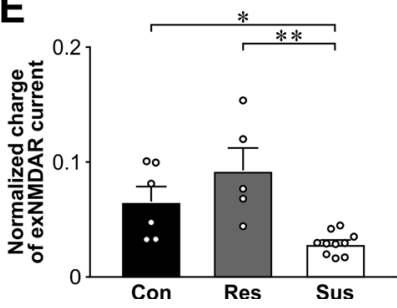

Fig. 2 Synaptic and extrasynaptic NMDAR function in the hippocampal CA1 region of control, resilient, and susceptible mice. a Schematic diagrams on the left show typical paths of susceptible and resilient mice during a social interaction test with the CD1 mouse present in the enclosure. Social interaction (SI) was examined $24 \mathrm{~h}$ after the last social defeat session. SI ratio = [time spent in the interaction zone (gray) when a CD1 mouse was in the enclosure]/ [time spent in the interaction zone when the enclosure was empty (not shown)]. Right: Histograms of the SI ratio. Non-stressed control (Con): $1.80 \pm 0.24 \mathrm{~s}(n=16)$ vs. Resilient (Res): $1.63 \pm 0.12 \mathrm{~s}(n=17)$ $p=0.627$. Con vs. Susceptible (Sus): $0.53 \pm 0.07 \mathrm{~s}(n=18) ; p=2.03 \mathrm{E}-$ 05 . Res vs. Sus; $p=4.82 \mathrm{E}-07$. Post hoc Wilcoxon tests after Kruskal-Wallis test. Mean $\pm \mathrm{SEM},{ }^{* * *} p<0.001$. b Left: Representative traces of evoked field excitatory postsynaptic potentials (fEPSPs) that were recorded from hippocampal slices of control, resilient, and susceptible mice. Currents used for evoked fEPSPs were adjusted to recruit similar levels of presynaptic activation, which was represented by the fiber volley $(f v)$ amplitude. Scale bar $=0.1 \mathrm{mV}, 5 \mathrm{~ms}$. Right: Scatter plots show the relationship between the slope of NMDAR fEPSPs and $f v$ amplitude in control $(n=9$ recordings/3 mice), resilient ( $n=11$ recordings/4 mice), and susceptible mice ( $n=11$ recordings $/ 4$ mice); $p=0.621$, group effect after repeated measures ( $f v$ amplitude) ANOVA. Mean \pm SEM. c Representative traces of sNMDAR before MK801 blockade and TBOA-induced exNMDAR currents from hippocampal slices of control (left), resilient (middle) and susceptible mice (right). Scale bar $=50 \mathrm{pA}, 500 \mathrm{~ms}$. d Histograms of the amplitude of TBOA-induced exNMDAR currents that were normalized by the amplitude of sNMDAR currents. Con: $0.175 \pm 0.033$ ( $n=6$ neurons/ 6 mice) vs. Sus: $0.083 \pm 0.009(n=10$ neurons/10 mice); $p=0.011$. Res: $0.215 \pm 0.050$ ( $n=5$ neurons $/ 5$ mice) vs. Sus; $p=0.006$. Post hoc Wilcoxon tests after Kruskal-Wallis test. Mean $\pm \mathrm{SEM},{ }^{*} p<0.05,{ }^{* *} p<0.01$. e Histograms of the charge of TBOA-induced exNMDAR currents that were normalized by the charge of sNMDAR currents. Con: $0.066 \pm 0.013$ ( $n=6$ neurons/ 6 mice) vs. Sus: $0.029 \pm 0.003$ ( $n=10$ neurons $/ 10$ mice); $p=0.011$. Res: $0.093 \pm 0.020$ ( $n=5$ neurons $/ 5$ mice) vs. Sus; $p=0.004$. Post hoc Wilcoxon tests after Kruskal-Wallis test. Mean \pm SEM, ${ }^{*} p<0.05,{ }^{* *} p<$ 0.01

currents, these currents were insensitive to the puff application of Mem-GNP (Fig. 4d). These findings suggest that GNPs could reach extrasynaptic, but not synaptic locations.

We next synthesized GNPs that were coated with an NMDAR agonist TZG. We found that TZG-coated GNPs (TZG-GNP) induced a whole-cell inward current that can be abolished by MK801 (Fig. 4e), suggesting that exNMDARs were activated by TZG-GNP. To find out if TZG-GNP selectively targets exNMDARs, we examined the effect of TZG-GNP and NMDA on electrically evoked sNMDAR currents. We hypothesized that puffing NMDA in slices would induce activation of NMDARs in both synaptic and extrasynaptic compartments, which would reduce the amplitude of electrically evoked sNMDAR currents. During the puffing of TZG-GNP $(160 \mathrm{nM})$ and NMDA $(10 \mu \mathrm{M})$ to induce a similar increase in whole-cell currents (Fig. 4f), we found that electrically evoked sNMDAR currents were reduced during NMDA application only. These findings support the selectivity of TZG-GNP to activate exNMDARs. Finally, we tested the effect of drug free-GNP, TZGGNP and Mem-GNP on the SI ratio of control and defeated mice. We found that all these GNPs did not affect the SI ratio of control mice (Fig. 4g). While defeated mice that were infused with drug free-GNP or Mem-GNP displayed social avoidance, TZG-GNP prevented social avoidance behavior in defeated mice. In parallel to findings from the NAC experiments, these data strongly support a notion that exNMDAR activation prevents social avoidance behavior in mice after CSDS.

The fast-acting antidepressant ketamine, an NMDAR antagonist, has been shown to prevent social avoidance behavior after CSDS [13]. Since our findings support the importance of exNMDARs in stress resilience, ketamine may protect exNMDARs function by 
A

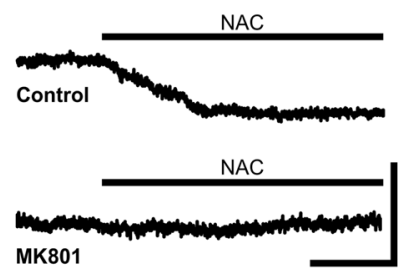

C
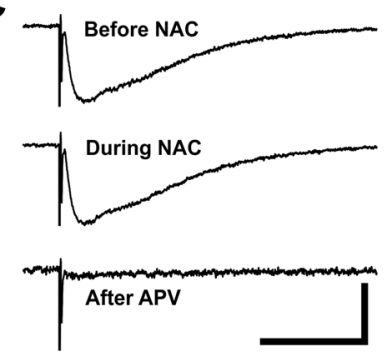

D
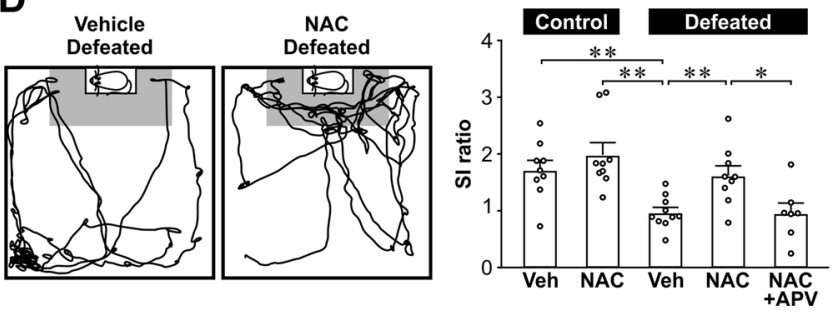

Fig. 3 Effect of $\mathrm{N}$-acetylcysteine (NAC) on NMDAR function and social interaction of defeated mice. a Representative traces of NAC $(100 \mu \mathrm{M})$-induced whole-cell currents recorded from a CA1 neuron. Recordings were performed under control conditions (top) or during MK801 blockade $(40 \mu \mathrm{M}$, bottom). Scale bar $=100 \mathrm{pA}, 3$ min. b Histograms of NAC-induced whole-cell currents without (control) or with the presence of NMDAR antagonists (MK801 at 40 $\mu \mathrm{M}$ or APV at $100 \mu \mathrm{M})$. Control: $36.3 \pm 7.6 \mathrm{pA}(n=4$ neurons/4 mice) vs. MK801: $0.88 \pm 1.01 \mathrm{pA}(n=3$ neurons $/ 3$ mice); $p=0.008$. Control vs. APV: $10.9 \pm 4.98 \mathrm{pA}$ ( $n=4$ neurons $/ 3$ mice); $p=0.029$. Post hoc Tukey's test after ANOVA. Mean \pm SEM, ${ }^{*} p<0.05,{ }^{* *} p<0.01$. c Left: Representative traces of evoked field excitatory postsynaptic potentials (fEPSPs) that were recorded before (top) and during NAC treatment $(100 \mu \mathrm{M}$; middle). Note that synaptic NMDAR (sNMDAR)-mediated fEPSPs were completely abolished by an NMDAR antagonist APV $(50 \mu \mathrm{M}$, bottom). Right: Scatter plots show the effect of NAC treatment $(100 \mu \mathrm{M}$, black bar) on the slope of NMDAR fEPSPs ( $n=5$ recordings $/ 3$ mice). Mean \pm SEM. d Left: Schematic diagrams show examples of moving paths of two mice which have received intrahippocampal infusions of either vehicle or NAC before the social interaction (SI) test. A CD1 mouse was present in the enclosure. Right: Histograms show the $\mathrm{SI}$ ratio of control and defeated mice that have received intrahippocampal infusions $(0.5 \mu \mathrm{l} / \mathrm{side})$ of vehicle, NAC $(100 \mathrm{mM})$, or NAC + APV $(5 \mathrm{mM}) 30 \mathrm{~min}$ before the SI test. Control vehicle: $1.71 \pm 0.17$ (Con Veh; $n=9$ ) vs. defeated vehicle: $0.95 \pm 0.17$ (Def Veh; $n=10$ ); $p=0.005$. Control NAC: $1.98 \pm 0.21$ (Con NAC; $n=9$ ) vs. Def Veh; $p=5.02 \mathrm{E}-04$. Defeated NAC: $1.62 \pm 0.17$ (Def NAC; $n=9$ ) vs. Def Veh; $p=0.006$. Def NAC vs. Defeated NAC + APV: $0.95 \pm 0.18$ (Def NAC + APV; $n=7$ ); $p=0.026$ Post hoc Wilcoxon tests after Kruskal-Wallis test. Mean $\pm \mathrm{SEM},{ }^{*} p<$ $0.05,{ }^{* *} p<0.01$

preferentially blocking NMDARs in synapses. In parallel with this proposal (Fig. 5a), we found that the percent blockade of the amplitude (Fig. 5b) and the charge (Fig. 5c) of sNMDARs were always higher than that of exNMDARs under all ketamine concentrations tested. Our findings therefore suggest that the sparing of exNMDARs or the increase in exNMDAR vs. SNMDAR
A

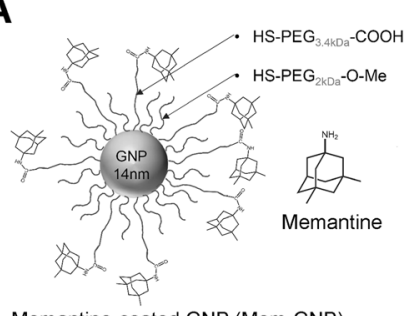

B NMDA

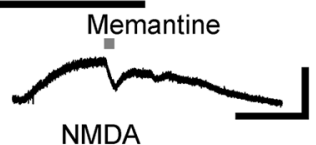

NMDA

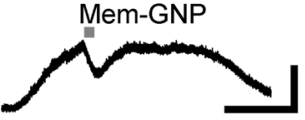

Memantine-coated GNP (Mem-GNP)
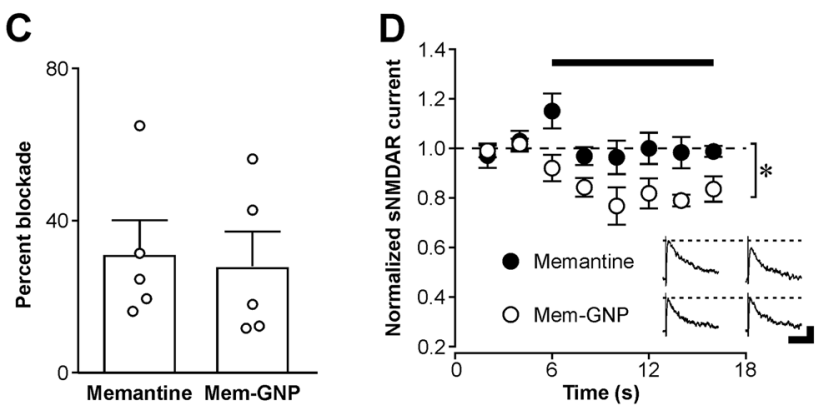

E
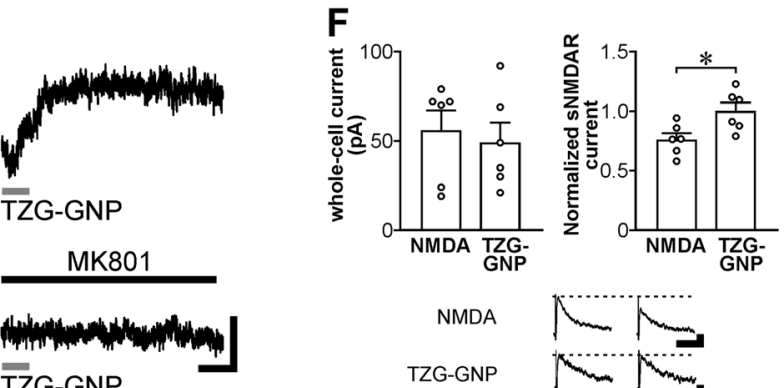

NMDA

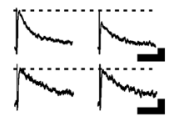

$\mathbf{G}$

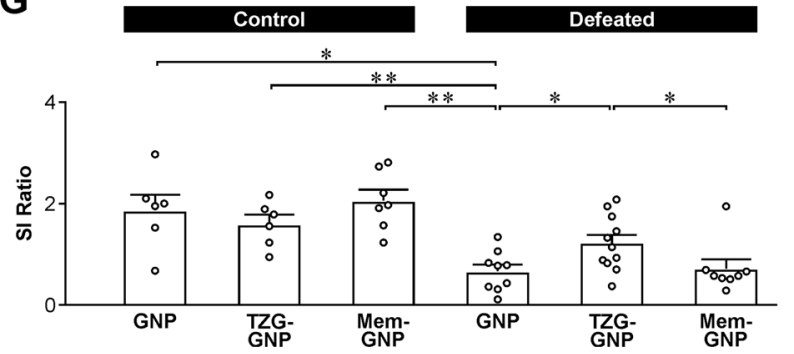

ratio could be responsible for the antidepressant action of ketamine.

\section{DISCUSSION}

We provide several lines of evidence to support the importance of hippocampal exNMDAR function on the susceptibility to CSDS. We found that after blocking SNMDAR currents with MK801, both TBOA- and tetanus-induced exNMDAR function in susceptible mice were lower than that in resilient and non-stressed control mice. Susceptible mice also exhibited lower APV-mediated blockade of whole-cell currents, which correspond to the blockade of exNMDAR currents due to ambient levels of glutamate, than resilient mice. Notably, no differences in sNMDAR function between these mouse groups were found. NAC, which preferentially activates exNMDAR via astrocytic cystine-glutamate antiporters, prevented a decrease in the SI ratio of defeated mice in a NMDAR-dependent manner. Although NAC is also known for its roles other than facilitating exNMDAR function, such as being an antioxidant precursor to glutathione and activating kinases such 
Fig. 4 Intrahippocampal infusion of NMDAR agonist-coated gold nanoparticles ameliorated the impact of social defeat on social interaction. a A schematic diagram of memantine-coated gold nanoparticle (Mem-GNP). Memantine (shown in the inset) was coated on GNP with a diameter of $14 \mathrm{~nm}$ diameter to create MemGNP with a hydrodynamic radius of around $35 \mathrm{~nm}$. b Representative traces of currents induced by the bath application of NMDA $(10 \mu \mathrm{M}$, black bars), an agonist that activates both synaptic and extrasynaptic NMDAR currents. Gray bars represent the puff application of either free memantine $(10 \mu \mathrm{M}$, above) or Mem-GNP $(60 \mathrm{nM}$, below). Scale bar $=100 \mathrm{pA}, 1 \mathrm{~min}$. c Histograms show the blockade of NMDA-induced currents by either free memantine $(n=5$ neurons/4 mice) or Mem-GNP ( $n=5$ neurons $/ 5$ mice) in panel b. Mean \pm SEM. d Scatter plots show the blockade of sNMDAR currents during the puff application (black bar) of either free memantine (black circles, $n=5$ neurons/4 mice) or Mem-GNP (white circles, $n=6$ neurons $/ 4$ mice). ${ }^{*} p<0.05$, group effect after repeated measures (time after agonist/antagonist application) ANOVA. Mean \pm SEM. Inset shows representative traces of evoked sNMDAR currents before (left) and during antagonist treatment (right). Traces on top and bottom correspond to the effect of free memantine and Mem-GNP, respectively, on sNMDAR currents. Dashed lines represent the level of sNMDAR current before antagonist application. Scale bar $=40 \mathrm{pA}, 200 \mathrm{~ms}$. e Representative traces of whole-cell currents induced by the puff application of TZG-GNP (gray bars). The trace below was recorded from the same neurons above but during the bath perfusion of MK801. Percent blockade of TZG-GNP induced currents by MK801 from four experiments $=80.0 \pm 2.8 \%$ (mean \pm SEM). Scale bar $=25 \mathrm{pA}, 4 \mathrm{~s}$. $f$ Top left: Histograms show the wholecell currents induced by either NMDA $(10 \mu \mathrm{M}, n=6$ neurons $/ 2$ mice $)$ or TZG-GNP ( $160 \mathrm{nM}, n=6$ neurons/5 mice) during the recording of evoked SNMDAR currents. Top right: Histograms show the amplitude of evoked sNMDAR currents during NMDA ( $n=6$ neurons/2 mice) or TZG-GNP application ( $n=6$ neurons/5 mice). These sNMDAR currents were normalized by their amplitudes before agonist application. Student's $t$-test, $p=0.021$. Mean \pm SEM, ${ }^{* *} p<$ 0.05 . Bottom: Representative traces of evoked sNMDAR currents before (left) and during agonist treatment (right). Traces on top and bottom correspond to the effect of NMDA and TZG-GNP, respectively, on sNMDAR currents. Dashed lines represent the level of sNMDAR current before antagonist application. Scale bar $=40 \mathrm{pA}$, $200 \mathrm{~ms}$. g Histograms summarize the social interaction ratio (SI ratio) of control and defeated mice that have received intrahippocampal infusion ( $60 \mathrm{nM}, 0.5 \mu \mathrm{l} / \mathrm{side}$ ) of non-coated GNP, TZG-GNP or MemGNP 30 min before the SI test. Control non-coated GNP: $1.87 \pm 0.31$ (Control GNP; $n=6$ ) vs. Defeated non-coated GNP: $0.67 \pm 0.13$ (Defeated GNP; $n=9) ; p=0.011$. Control TZG-GNP: $1.60 \pm 0.18(n=$ 6) vs. Defeated GNP; $p=0.006$. Control Mem-GNP: $2.06 \pm 0.22(n=7)$ vs. Defeated GNP; $p=0.002$. Defeated TZG-GNP: $1.22 \pm 0.16(n=11)$ vs. Defeated GNP; $p=0.017$. Defeated TZG-GNP vs. Defeated TZGMem: $0.72 \pm 0.18(n=8) ; p=0.012$. Post hoc Wilconxon tests after ANOVA. Mean \pm SEM, ${ }^{*} p<0.05,{ }^{* *} p<0.01$

as ERK and MAPK [28], we found that activating exNMDARs by TZG-GNP that cannot enter the synaptic cleft enhanced the SI ratio of defeated mice. Interestingly, ketamine, a fast-acting antidepressant, preferentially inhibited sNMDARs, but not exNMDARs. Given the importance of using CSDS to model anxiety- and depression-related phenotypes, exNMDARs may be novel molecular targets for treating anxiety and depression.

Using inbred mice with different susceptibilities to CSDS has been shown to be a highly useful approach to determine biological mechanisms underlying depression. Mice that are susceptible to CSDS by showing social avoidance in the SI test also display depression-related behaviors such as anhedonia, behavioral despair, and sleep disturbance [9-11]. In addition, depression-related behaviors in susceptible mice can be rescued by chronic but not acute antidepressant treatments [12]. However, caveats of this approach include the use of a short SI test, which could be subjected to errors, such as differences in the testing parameters (e.g. lighting, acoustic, and context in the testing room) and in personnel training. Social avoidance can be found in other psychiatric disorders such as attention deficit hyperactivity disorder, autism spectrum disorder, and schizophrenia. The separation of defeated animals into either susceptible or resilient groups also overlooks the quantitative relationship between the SI ratio and the studied parameters. Finally, whether targeting exNMDARs could rescue other depression- and anxiety-related behaviors of this model remains to be examined in future.

The susceptibility to CSDS not only involves brain regions including the nucleus accumbens and prefrontal cortex, but also the hippocampus. Individual differences in stress susceptibility in rodents have been associated with changes in gene [29] and protein expression in the hippocampus [30]. Furthermore, the susceptibility to CSDS can be abolished by inhibiting hippocampal neurogenesis [31]. Using a longitudinal MRI approach [20], we have shown differences in the developmental trajectory of hippocampal volume in mice that were susceptible or resilient to CSDS. Lastly, a recent optogenetic study has revealed the importance of the ventral hippocampal projection to the nucleus accumbens in the susceptibility to CSDS [32]. Various findings suggest that the dorsal hippocampus regulates the vulnerability and expression of depression-related behaviors after stress. For instance, inhibiting cholinesterase activity or expression in the dorsal hippocampus alone can induce various types of depressionrelated behaviors [33]. The susceptibility to CSDS has been associated with alterations in microRNA expression [34] and microstructural alterations in the dorsal hippocampus [16]. One plausible mechanism underlying the contribution of the dorsal hippocampus to depression-related behaviors is an increased ratio of excitation/inhibition in this region. Chronic stress-related decrease in the function of interneurons has been associated with increased depression-related behaviors [35]. A decrease in neuroligin 2 expression, which is known for its roles in inhibitory synapse formation, was associated with stress-induced deficits in sociability and aggression [36]. Our findings further suggest that dorsal hippocampal function could be regulated by exNMDARs to affect the susceptibility to CSDS.

While exNMDARs are known for their roles in neuronal death [3], emerging findings also supported their physiological roles. exNMDARs can be activated by the repetitive stimulation of synaptic inputs [37], astrocytic release of glutamate [38], or by ambient levels of glutamate [26]. exNMDAR activation could produce a persistent depolarization that lasts for seconds [39], which in turn enhances neuronal excitability and modulates the computation of synaptic signals [37]. exNMDARs can also be regulated by stress. The inhibition of glutamate uptake by stress [40] could facilitate exNMDAR function and long-term depression (LTD [41]) by increasing extracellular levels of glutamate. The mobility of exNMDARs can also be decreased by the stress hormone corticosterone, although its effect on reducing sNMDARs' mobility could be more prominent [42]. sNMDARs and exNMDARs may play distinct roles in regulating brain functions. While long-term potentiation and LTD can be produced by high- and low-frequency stimulation of sNMDARs, activation of exNMDARs alone or together with sNMDARs (e.g. by NMDA) induces LTD only [43]. Interestingly, these two receptor pools could interact with each other to regulate neuronal function, so that while the activation of exNMDARs alone enhances an $h$ channel conductance, this conductance is reduced by the coactivation of sNMDARs and exNMDARs [44].

Previous studies have shown that social avoidance behavior during SI test after CSDS can be substantially reduced by altering the status (using an anesthetized CD1 mouse [9]) or the strain (using a C57 mouse [45]) of the social target in the enclosure, suggesting the importance of cognitive processing of defeatrelated information in the expression of this depression-related behavior. We propose that low exNMDAR function could affect the processing of defeat-related information in the hippocampus of 
A
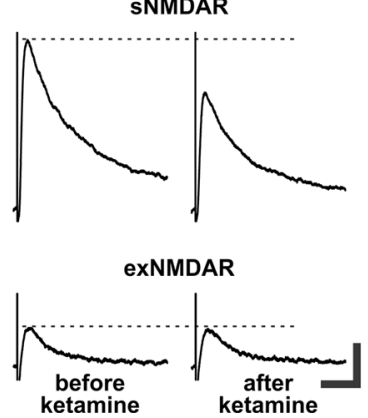

B

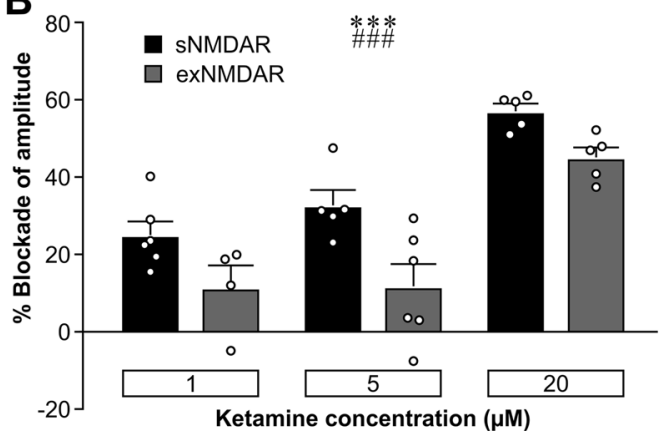

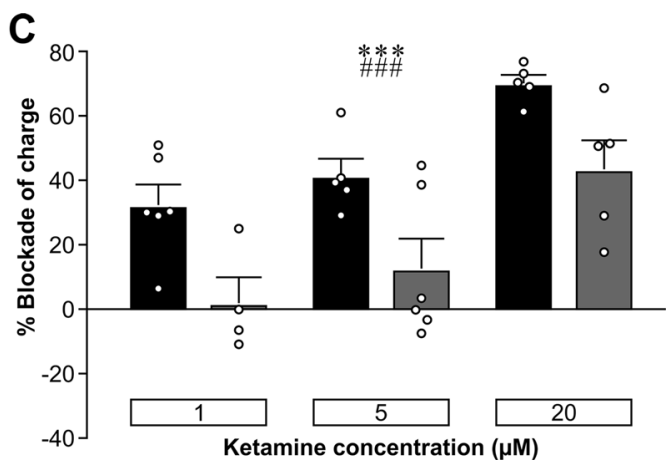

Fig. 5 The effect of ketamine on synaptic and extrasynaptic NMDAR function. a Representative traces of synaptic (sNMDAR, top) and extrasynaptic (exNMDAR, bottom) currents before and after ketamine treatment $(5 \mu \mathrm{M})$. Scale bar $=25 \mathrm{pA}, 100 \mathrm{~ms}$. b Histograms show the percent blockade of the amplitude of sNMDARs and exNMDARs by ketamine in different concentrations. sNMDAR: $1 \mu$ M ketamine, $n=6$ neurons/4 mice; $5 \mu \mathrm{M}$ ketamine, $n=5$ neurons/4 mice; $20 \mu \mathrm{M}$ ketamine, $n=5$ neurons $/ 4$ mice. exNMDAR: $1 \mu \mathrm{M}$ ketamine, $n=4$ neurons $/ 4$ mice; $5 \mu \mathrm{M}$ ketamine, $n=6$ neurons $/ 6$ mice; $20 \mu \mathrm{M}$ ketamine, $n=5$ neurons $/ 5$ mice). Simple effect tests (effect of synaptic location ( ${ }^{* * *} p<$ $0.001): p=1.61 \mathrm{E}-04$; effect of ketamine concentration ( $\left.{ }^{\# \#} p<0.001\right): p=6.30 \mathrm{E}-08$; synaptic location and ketamine interaction: $\left.p=0.529\right)$ were performed after a two-way ANOVA. Mean \pm SEM. c Histograms show the percent blockade of the charge of sNMDARs and exNMDARs by ketamine in different concentrations. sNMDAR: $1 \mu \mathrm{M}$ ketamine, $n=6$ neurons $/ 4$ mice; $5 \mu \mathrm{M}$ ketamine, $n=5$ neurons $/ 4$ mice; $20 \mu \mathrm{M}$ ketamine, $n=5$ neurons/4 mice. exNMDAR: $1 \mu \mathrm{M}$ ketamine, $n=4$ neurons $/ 4$ mice; $5 \mu \mathrm{M}$ ketamine, $n=6$ neurons $/ 6$ mice; $20 \mu \mathrm{M}$ ketamine, $n=5$ neurons/ 5 mice). Simple effect tests (effect of synaptic location ( $\left.{ }^{* * *} p<0.001\right): p=7.42 \mathrm{E}-05$; effect of ketamine concentration (\#\#\# $p<0.001$ ): $p=4.71 \mathrm{E}-05$; synaptic location and ketamine interaction: $p=0.970$ ) were performed after a two-way ANOVA. Mean \pm SEM

susceptible mice. exNMDAR activation has been suggested to function as a dynamic range compressor to suppress strong stimuli [37]. Low exNMDAR function in susceptible mice may impair this signal filtering process and facilitate the encoding of defeat-related information. Consolidation and retrieval of defeatrelated information may also be enhanced by low exNMDAR function, since these receptors are important for LTD [43] that mediates forgetting [46] and impairs memory retrieval [47]. Indeed, our findings that activating exNMDARs by NAC or TZGGNP neutralized the effects of CSDS on SI may suggest the involvement of LTD processes to disrupt the retrieval of defeatrelated memory. Notably, all exNMDAR-modulating drugs we have tested did not affect social behavior of non-stressed mice, suggesting these extrasynaptic receptors do not directly mediate the expression of social behavior, but instead regulate the behavioral effects of stress on sociability. Finally, exNMDARs have been associated with signaling pathways that could lead to antidepressant actions. exNMDARs can activate Forkhead box $\mathrm{O}$ (FoxO) [3]. Interestingly, knocking down FoxO1 signaling enhances depression-related behavior [48]. Activation of exNMDARs could also induce calpain-dependent cleavage of striatal-enriched protein tyrosine phosphatase (STEP [49]), which is in parallel to the emerging antidepressant effects of STEP inhibitors [50].

We show that ketamine, a highly promising antidepressant for its fast-acting therapeutic effect and its efficacy in treatmentresistant depression [5], blocks sNMDARs more effectively than exNMDARs. Whether NMDAR blockade is necessary for the antidepressant effect of ketamine remains controversial. The antidepressant effects of ketamine could be related to their non-NMDAR targets [51] (but also see [52]). While ketamine is an NMDAR antagonist, antidepressant effects can also be produced by enhancing NMDAR function via glycine transporter inhibitors [53] or partial agonists such as D-cycloserine [54]. Finally, some NMDAR antagonists like memantine fail to produce antidepressant effect [55]. Based on our findings, we propose that the antidepressant effects of NMDAR-targeting drugs are triggered by either sparing exNMDAR function or by increasing the ratio of exNMDAR vs. sNMDAR function as with ketamine, but not by memantine that at low concentrations primarily targets exNMDARs $[23,56]$. Notably, findings from the current study and others [25] strongly suggest that both GluN2A- and GluN2B- containing NMDARs can be found in synaptic and extrasynaptic locations in the hippocampus of adult rodents. Antidepressant effects of GluN2B antagonists [57] therefore are likely not exclusively due to their blockade of exNMDARs. Examining the impact of NMDAR antagonists on sNMDARs and exNMDARs could provide a clearer picture of how antidepressant effects are generated.

Since we examined exNMDAR function in defeated mice after stress exposure, it remains unclear if innate differences in exNMDAR function predict their susceptibility to CSDS. Using longitudinal imaging approaches, we [20] and others [16] have revealed differences in hippocampal volume and microstructures between susceptible and resilient mice before stress. In addition, employing the performance of a genetically diverse strain in a non-stressful short-term memory task to predict stress susceptibility, differences in hippocampal expression of AMPA receptors were found between potentially susceptible and resilient mice without stress exposure [14]. Future studies could develop PET tracers that label specifically exNMDARs in the brain to longitudinally compare the levels of these receptors before and after stress exposure in susceptible and resilient mice.

The role of exNMDARs in stress susceptibility could have important implications on the pathogenesis of depression in humans. Proton magnetic resonance spectroscopy studies revealed that glutamate levels in the MPFC [58], the cingulate cortex [59], the amygdala and the hippocampus [60] of depressed patients are lower than that in controls. This could be related to a reduced number of astrocytes [6], which determine extracellular concentrations of neurotransmitters including glutamate. Low glutamate levels could mediate depression symptoms by impairing exNMDAR activation. Interestingly, antidepressants such as ketamine could increase extracellular levels of glutamate [61], which would facilitate exNMDAR activation and so reduce depression-related symptoms. NAC, which facilitates exNMDAR activation, has been shown to ameliorate depressive symptoms [62]. Interestingly, a recent study showed that depression-related behaviors induced in $\mathrm{C} 57$ mice by chronic restraint stress are related to the lower expression of cystine-glutamate antiporter in the ventral hippocampus, which can be rescued by NAC treatments [63]. Targeting exNMDARs, by using TZG-GNP for example, could be a novel approach for treating depression. 


\section{FUNDING AND DISCLOSURE}

This work is supported by the Canadian Institutes of Health Research (137003). The authors declare no competing interests.

\section{ADDITIONAL INFORMATION}

Supplementary Information accompanies this paper at (https://doi.org/10.1038/ s41386-019-0325-8).

Publisher's note: Springer Nature remains neutral with regard to jurisdictional claims in published maps and institutional affiliations.

\section{REFERENCES}

1. Beneyto $M$, Kristiansen LV, Oni-Orisan A, McCullumsmith RE, Meador-Woodruff $\mathrm{JH}$. Abnormal glutamate receptor expression in the medial temporal lobe in schizophrenia and mood disorders. Neuropsychopharmacology. 2007;32:1888-902.

2. Iadarola ND, Niciu MJ, Richards EM, Vande Voort JL, Ballard ED, Lundin NB, et al. Ketamine and other N-methyl-D-aspartate receptor antagonists in the treatment of depression: a perspective review. Ther Adv Chronic Dis. 2015;6:97-14.

3. Hardingham GE, Bading $H$. Synaptic versus extrasynaptic NMDA receptor signalling: implications for neurodegenerative disorders. Nat Rev Neurosci. 2010;11:682-96.

4. Parsons MP, Raymond LA. Extrasynaptic NMDA receptor involvement in central nervous system disorders. Neuron. 2014;82:279-93.

5. Krystal JH, Sanacora G, Duman RS. Rapid-acting glutamatergic antidepressants: the path to ketamine and beyond. Biol Psychiatry. 2013;73:1133-41.

6. Rajkowska G, Stockmeier CA. Astrocyte pathology in major depressive disorder: insights from human postmortem brain tissue. Curr Drug Targets. 2013;14:1225-36.

7. Kang HJ, Voleti B, Hajszan T, Rajkowska G, Stockmeier CA, Licznerski $P$, et al. Decreased expression of synapse-related genes and loss of synapses in major depressive disorder. Nat Med. 2012;18:1413-7.

8. Lima-Ojeda JM, Vogt MA, Pfeiffer N, Dormann C, Kohr G, Sprengel R, et al. Pharmacological blockade of GluN2B-containing NMDA receptors induces antidepressant-like effects lacking psychotomimetic action and neurotoxicity in the perinatal and adult rodent brain. Prog Neuropsychopharmacol Biol Psychiatry. 2013;45:28-33.

9. Krishnan V, Han MH, Graham DL, Berton O, Renthal W, Russo SJ, et al. Molecular adaptations underlying susceptibility and resistance to social defeat in brain reward regions. Cell. 2007;131:391-404.

10. Wells AM, Ridener E, Bourbonais CA, Kim W, Pantazopoulos $\mathrm{H}$, Carroll Fl, et al. Effects of chronic social defeat stress on sleep and circadian rhythms are mitigated by kappa-opioid receptor antagonism. J Neurosci. 2017;37:7656-68.

11. Iniguez SD, Flores-Ramirez FJ, Riggs LM, Alipio JB, Garcia-Carachure I, Hernandez $M A$, et al. Vicarious social defeat stress induces depression-related outcomes in female mice. Biol Psychiatry. 2018;83:9-17.

12. Berton O, McClung CA, Dileone RJ, Krishnan V, Renthal W, Russo SJ, et al. Essential role of BDNF in the mesolimbic dopamine pathway in social defeat stress. Science. 2006;311:864-8.

13. Donahue RJ, Muschamp JW, Russo SJ, Nestler EJ, Carlezon WA Jr.. Effects of striatal DeltaFosB overexpression and ketamine on social defeat stress-induced anhedonia in mice. Biol Psychiatry. 2014;76:550-8.

14. Schmidt MV, Trumbach D, Weber P, Wagner K, Scharf SH, Liebl C, et al. Individual stress vulnerability is predicted by short-term memory and AMPA receptor subunit ratio in the hippocampus. J Neurosci. 2010;30:16949-58.

15. Wagner KV, Marinescu D, Hartmann J, Wang XD, Labermaier C, Scharf SH, et al. Differences in FKBP51 regulation following chronic social defeat stress correlate with individual stress sensitivity: influence of paroxetine treatment. Neuropsychopharmacology. 2012;37:2797-808.

16. Liu X, Yuan J, Guang Y, Wang X, Feng Z. Longitudinal in vivo diffusion tensor imaging detects differential microstructural alterations in the hippocampus of chronic social defeat stress-susceptible and resilient mice. Front Neurosci. 2018;12:613.

17. Ma $X$, Wu $Y$, Jin $S$, Tian $Y$, Zhang $X$, Zhao $Y$, et al. Gold nanoparticles induce autophagosome accumulation through size-dependent nanoparticle uptake and lysosome impairment. ACS Nano. 2011;5:8629-39.

18. Savchenko A, Braun GB, Molokanova E. Nanostructured antagonist of extrasynaptic NMDA receptors. Nano Lett. 2016;16:5495-502.

19. Savtchenko LP, Rusakov DA. The optimal height of the synaptic cleft. Proc Natl Acad Sci USA. 2007;104:1823-8.

20. Tse YC, Montoya I, Wong AS, Mathieu A, Lissemore J, Lagace DC, et al. A longitudinal study of stress-induced hippocampal volume changes in mice that are susceptible or resilient to chronic social defeat. Hippocampus. 2014;24:1120-8.
21. Peartree NA, Hood LE, Thiel KJ, Sanabria F, Pentkowski NS, Chandler KN, et al. Limited physical contact through a mesh barrier is sufficient for social rewardconditioned place preference in adolescent male rats. Physiol Behav. 2012;105:749-56.

22. Tse YC, Bagot RC, Hutter JA, Wong AS, Wong TP. Modulation of synaptic plasticity by stress hormone associates with plastic alteration of synaptic NMDA receptor in the adult hippocampus. PLoS ONE 2011;6:e27215.

23. Milnerwood AJ, Gladding CM, Pouladi MA, Kaufman AM, Hines RM, Boyd JD, et al. Early increase in extrasynaptic NMDA receptor signaling and expression contributes to phenotype onset in Huntington's disease mice. Neuron. 2010;65:178-90.

24. Cull-Candy S, Brickley S, Farrant M. NMDA receptor subunits: diversity, development and disease. Curr Opin Neurobiol. 2001;11:327-35.

25. Harris AZ, Pettit DL. Extrasynaptic and synaptic NMDA receptors form stable and uniform pools in rat hippocampal slices. J Physiol. 2007;584:509-19.

26. Le Meur K, Galante M, Angulo MC, Audinat E. Tonic activation of NMDA receptors by ambient glutamate of non-synaptic origin in the rat hippocampus. J Physiol. 2007;580:373-83.

27. Berk M, Malhi GS, Gray LJ, Dean OM. The promise of N-acetylcysteine in neuropsychiatry. Trends Pharmacol Sci. 2013;34:167-77.

28. Zafarullah M, Li WQ, Sylvester J, Ahmad M. Molecular mechanisms of Nacetylcysteine actions. Cell Mol Life Sci. 2003;60:6-20.

29. Bergstrom A, Jayatissa MN, Thykjaer T, Wiborg O. Molecular pathways associated with stress resilience and drug resistance in the chronic mild stress rat model of depression: a gene expression study. J Mol Neurosci. 2007;33:201-15.

30. Heinzmann JM, Thoeringer CK, Knapman A, Palme R, Holsboer F, Uhr M, et al. Intrahippocampal corticosterone response in mice selectively bred for extremes in stress reactivity: a microdialysis study. J Neuroendocrinol. 2010;22:1187-97.

31. Lagace DC, Donovan MH, DeCarolis NA, Farnbauch LA, Malhotra S, Berton O, et al. Adult hippocampal neurogenesis is functionally important for stress-induced social avoidance. Proc Natl Acad Sci USA. 2010;107:4436-41.

32. Bagot RC, Parise EM, Pena CJ, Zhang HX, Maze I, Chaudhury D, et al. Ventral hippocampal afferents to the nucleus accumbens regulate susceptibility to depression. Nat Commun. 2015;6:7062.

33. Mineur YS, Obayemi A, Wigestrand MB, Fote GM, Calarco CA, Li AM, et al. Cholinergic signaling in the hippocampus regulates social stress resilience and anxiety- and depression-like behavior. Proc Natl Acad Sci USA. 2013;110:3573-8.

34. Munoz-Llanos M, Garcia-Perez MA, Xu X, Tejos-Bravo M, Vidal EA, Moyano TC, et al. MicroRNA profiling and bioinformatics target analysis in dorsal hippocampus of chronically stressed rats: relevance to depression pathophysiology. Front Mol Neurosci. 2018;11:251.

35. Riga D, Kramvis I, Koskinen MK, van Bokhoven $\mathrm{P}$, van der Harst JE, Heistek TS et al Hippocampal extracellular matrix alterations contribute to cognitive impairment associated with a chronic depressive-like state in rats. Sci Transl Med. 2017;9: eaai8753.

36. van der Kooij MA, Fantin M, Kraev I, Korshunova I, Grosse J, Zanoletti O, et al. Impaired hippocampal neuroligin-2 function by chronic stress or synthetic peptide treatment is linked to social deficits and increased aggression. Neuropsychopharmacology. 2014;39:1148-58.

37. Oikonomou KD, Short SM, Rich MT, Antic SD. Extrasynaptic glutamate receptor activation as cellular bases for dynamic range compression in pyramidal neurons. Front Physiol. 2012;3:334.

38. Shigetomi E, Bowser DN, Sofroniew MV, Khakh BS. Two forms of astrocyte calcium excitability have distinct effects on NMDA receptor-mediated slow inward currents in pyramidal neurons. J Neurosci. 2008;28:6659-63.

39. Suzuki T, Kodama S, Hoshino C, Izumi T, Miyakawa H. A plateau potential mediated by the activation of extrasynaptic NMDA receptors in rat hippocampal CA1 pyramidal neurons. Eur J Neurosci. 2008;28:521-34.

40. Zink M, Vollmayr B, Gebicke-Haerter PJ, Henn FA. Reduced expression of glutamate transporters vGluT1, EAAT2 and EAAT4 in learned helpless rats, an animal model of depression. Neuropharmacology. 2010;58:465-73.

41. Yang $\mathrm{CH}$, Huang $\mathrm{CC}$, Hsu KS. Behavioral stress enhances hippocampal CA1 longterm depression through the blockade of the glutamate uptake. J Neurosci. 2005;25:4288-93.

42. Mikasova L, Xiong $H$, Kerkhofs A, Bouchet D, Krugers HJ, Groc L. Stress hormone rapidly tunes synaptic NMDA receptor through membrane dynamics and mineralocorticoid signalling. Sci Rep. 2017;7:8053.

43. Liu DD, Yang Q, Li ST. Activation of extrasynaptic NMDA receptors induces LTD in rat hippocampal CA1 neurons. Brain Res Bull. 2013;93:10-6.

44. Wu YW, Grebenyuk S, McHugh TJ, Rusakov DA, Semyanov A. Backpropagating action potentials enable detection of extrasynaptic glutamate by NMDA receptors. Cell Rep. 2012;1:495-505.

45. Venzala E, Garcia-Garcia AL, Elizalde N, Delagrange P, Tordera RM. Chronic social defeat stress model: behavioral features, antidepressant action, and interaction with biological risk factors. Psychopharmacology (Berl). 2012;224:313-25. 
46. Migues PV, Liu L, Archbold GE, Einarsson EO, Wong J, Bonasia K, et al. Blocking synaptic removal of GluA2-containing AMPA receptors prevents the natural forgetting of long-term memories. J Neurosci. 2016;36:3481-94.

47. Wong TP, Howland JG, Robillard JM, Ge Y, Yu W, Titterness AK, et al. Hippocampal long-term depression mediates acute stress-induced spatial memory retrieval impairment. Proc Natl Acad Sci USA. 2007;104:11471-6.

48. Polter A, Yang S, Zmijewska AA, Van GT, Paik JH, Depinho RA, et al. Forkhead box, class $O$ transcription factors in brain: regulation and behavioral manifestation. Biol Psychiatry. 2009;65:150-9.

49. Xu J, Kurup P, Zhang Y, Goebel-Goody SM, Wu PH, Hawasli AH, et al. Extrasynaptic NMDA receptors couple preferentially to excitotoxicity via calpain-mediated cleavage of STEP. J Neurosci. 2009;29:9330-43.

50. Kulikova E, Kulikov A. Striatal-enriched tyrosine protein phosphatase (STEP) in the mechanisms of depressive disorders. Curr Protein Pept Sci. 2017;18:1152-62.

51. Zanos P, Moaddel R, Morris PJ, Georgiou P, Fischell J, Elmer Gl, et al. NMDAR inhibition-independent antidepressant actions of ketamine metabolites. Nature. 2016;533:481-6.

52. Suzuki K, Nosyreva E, Hunt KW, Kavalali ET, Monteggia LM. Effects of a ketamine metabolite on synaptic NMDAR function. Nature. 2017;546:E1-3.

53. Huang $C C$, Wei $I H$, Huang $C L$, Chen $K T$, Tsai $M H$, Tsai $P$, et al. Inhibition of glycine transporter-I as a novel mechanism for the treatment of depression. Biol Psychiatry. 2013;74:734-41.

54. Heresco-Levy U, Gelfin G, Bloch B, Levin R, Edelman S, Javitt DC, et al. A randomized add-on trial of high-dose D-cycloserine for treatment-resistant depression. Int J Neuropsychopharmacol. 2013;16:501-6.

55. Gideons ES, Kavalali ET, Monteggia LM. Mechanisms underlying differential effectiveness of memantine and ketamine in rapid antidepressant responses. Proc Natl Acad Sci USA. 2014;111:8649-54.
56. Okamoto S, Pouladi MA, Talantova M, Yao D, Xia P, Ehrnhoefer DE, et al. Balance between synaptic versus extrasynaptic NMDA receptor activity influences inclusions and neurotoxicity of mutant huntingtin. Nat Med. 2009;15:1407-13.

57. Ibrahim L, Diaz GN, Jolkovsky L, Brutsche N, Luckenbaugh DA, Herring WJ, et al. A randomized, placebo-controlled, crossover pilot trial of the oral selective NR2B antagonist MK-0657 in patients with treatment-resistant major depressive disorder. J Clin Psychopharmacol. 2012;32:551-7.

58. Yildiz-Yesiloglu A, Ankerst DP. Review of $1 \mathrm{H}$ magnetic resonance spectroscopy findings in major depressive disorder: a meta-analysis. Psychiatry Res. 2006;147:1-25.

59. Auer DP, Putz B, Kraft E, Lipinski B, Schill J, Holsboer F. Reduced glutamate in the anterior cingulate cortex in depression: an in vivo proton magnetic resonance spectroscopy study. Biol Psychiatry. 2000;47:305-13.

60. Michael N, Erfurth A, Ohrmann P, Arolt V, Heindel W, Pfleiderer B. Neurotrophic effects of electroconvulsive therapy: a proton magnetic resonance study of the left amygdalar region in patients with treatment-resistant depression. Neuropsychopharmacology. 2003;28:720-5.

61. Lener MS, Niciu MJ, Ballard ED, Park M, Park LT, Nugent AC, et al. Glutamate and gamma-aminobutyric acid systems in the pathophysiology of major depression and antidepressant response to ketamine. Biol Psychiatry. 2017;81:886-97.

62. Deepmala SJ, Kumar N, Delhey L, Berk M, Dean O, et al. Clinical trials of Nacetylcysteine in psychiatry and neurology: a systematic review. Neurosci Biobehav Rev. 2015;55:294-21.

63. Nasca C, Bigio B, Zelli D, de Angelis $P$, Lau T, Okamoto $M$, et al. Role of the astroglial glutamate exchanger $\mathrm{xCT}$ in ventral hippocampus in resilience to stress. Neuron. 2017;96:402-13 e405. 